ESAIM: PROCEEDINGS AND SURVEYS, February 2015, Vol. 49, p. 53-64

Samira EL YACOUBI, Larbi AFIFI, El Hassan ZERRIK, Abdessamad TRIDANE, Editors

\title{
EPIDEMIC SPREADING AND RISK PERCEPTION IN MULTIPLEX NETWORKS: A SELF-ORGANIZED PERCOLATION METHOD*
}

\author{
E. MASSARO ${ }^{1}$ AND F. BAGNOLI ${ }^{2}$
}

\begin{abstract}
The contribution of this paper is twofold. First, we develop a self-organized method which is able the evaluate the percolation threshold of the SIS dynamics in general networks by comparing computational results with theoretical ones. Then, we apply this method to the case of risk perception in which the probability of being infected is reduced by a component which is function of the information about the disease. We then study the interplay between epidemic spreading and risk perception on multiplex networks. The system is represented by two layers: one representing the physical contacts and the other one the virtual contacts in which people exchange information and become aware of the disease. The final contribution of this paper is to comprehend the effectiveness of the source of information in modelling the risk perception in epidemic modelling: we found that the similarity between the physical and the information networks determine the possibility of stopping the infection for a sufficiently high precaution level: if the networks are too different there is no mean of avoiding the epidemics.
\end{abstract}

Résumé. La contribution de cet article est double. Tout d'abord, nous développons une méthode auto-organisée qui est en mesure d'évaluer le seuil de percolation de la dynamique SIS dans les réseaux généraux en confrontant les résultats de simulation aux résultats théoriques. Ensuite, nous appliquons cette méthode pour le cas de la perception du risque dans lequel la probabilité d'infection est réduite d'un élément qui est fonction de l'information sur la maladie. Nous étudions après l'interaction entre la propagation de l'épidémie et la perception du risque sur des réseaux multiplex. Le système est représenté par deux couches: une représentation des contacts physiques et l'autre les contacts virtuels dans lesquels les gens échangent des informations et prennent conscience de la maladie. La contribution finale de ce papier est de permettre la compréhension de l'efficacité de la source de l'information dans la représentation de la perception du risque pour la modélisation de l'épidémie. Nous avons constaté que la similitude entre les réseaux d'information et les réseaux physiques détermine la possibilité d'arrêter l'infection pour un niveau de précaution suffisamment élevé. En d'autres termes, si les réseaux sont trop différents il n'y a pas moyen d'éviter les épidémies.

\footnotetext{
* FB acknowledges partial financial support from the EU projects 288021 (EINS - Network of Excellence in Internet Science) and 611299 (SciCafe2.0). The authors are grateful to Dr. Nicola Perra for helpful discussions.

1 Risk and Decision Science Team, US Army Engineer Research and Development Center, 696 Virginia Rd., Concord, MA 01742, and Department of Civil and Environmental Engineering, Carnegie Mellon University, 5000 Forbes Ave, Pittsburgh, PA 15213; email emassaro@andrew.cmu.edu

2 Dipartimento di Fisica ed Astronomia and CSDC, Università degli Studi di Firenze, via G. Sansone 1, 50019 Sesto Fiorentino, Italy. Also INFN, Sez. di Firenze. email: franco.bagnoli@unifi.it
}

(C) EDP Sciences, SMAI 2015 


\section{INTRODUCTION}

In a previous work [1], some of us investigated the influence of the risk perception in epidemic spreading. We assumed that the knowledge about the diffusion of the disease among neighbours, without knowing who is actually infected, effectively lowers the probability of transmission, i.e., the effective infectiousness. We studied the worst case of an infection on a scale-free network with exponent $\gamma=2$ and we showed that in this case no degree of prevention is able to stop the infection and one has to take additional precaution for hubs, such as public officers and physicians.

We extend here the investigation to different network structures, on order to obtain a complete reference frame. For regular, random, Watts-Strogatz small-world and non-assortative scale-free networks with exponent $\gamma>3$ there is always a finite level of precaution parameter for which the epidemics go extinct. For scale-free networks with $\gamma<3$ the precaution level depends on the cut-off of the power-law, which at least depends on the finite number of the network.

We consider then an important factor of modern society: the fact that most of information comes no more from physical contacts nor from broadcasting media, but rather from the "virtual" social contact networks 2 -4]. A recent study, State of the news media for the United States [5], highlights this phenomena. It shows the extent of the influence of social networks, for what concerns subscribers who can read news published by newspapers. The $9 \%$ of the population claims to inquire "very often" through Facebook and Twitter and seven out of ten members are addressed to articles from newspapers and other sources by friends and family members.

We are therefore confronted with news coming mainly from an information network. On the other hand, the physical network of contacts is the environment where actual infections occur. We extend our model to the case in which the source of information, composed by mixed physical and virtual contacts, does not coincide with the actual source of infection, i.e., the physical contacts.

This system is well represented as a multiplex network 6 10, i.e., a graph composed by several layers in which the same set of $N$ nodes can be connected to each other by means of links belonging to different layers, which represents a specific case of interdependent network [11,12]. Recently, Granell et al. [13] have pointed out the attention to an interesting scenario where the multiplex corresponds to a two-layers network, one where the dynamics of the awareness about the disease, i.e., the information dynamics, evolves and another where the epidemic process spreads.

The first layer represents the information network where people become aware of the epidemic thanks to the news coming from virtual and physical contacts in various proportions. The second layer represents the physical contact network where the epidemic spreading takes place. While the news coming from real contacts actually carry information about the risk of being infected from neighbours, those from the virtual network are mainly useless, carrying at most information about the average penetration of the infection in the population.

In this paper we want to investigate the consequences of this discrepancy. We study how the percolation threshold of a susceptible-infected-susceptible (SIS) mode depends on the perception of the risk that affects the invectiveness probability when this information comes from the same contact network of the disease or from a different network. In other words, we study the interplay between risk perception and disease spreading on multiplex networks.

We are interested in the epidemic threshold, which is a quantity that it is not easily obtained from numerical simulations, for varying values of the parameters. We develop an extension of a self-organized formulation of percolation phenomena 14] that allows to obtain this threshold in just one simulation, for a sufficiently large system.

\section{THE NETWORK MODEL}

In this section we show the method we employed for generating multiplex networks. We start describing the method for generating regular, random and scale-free networks.

Let us denote by $a_{i j}=0,1$ the adjacency matrix of the network, $a_{i j}=1$ if there is a link from $j$ to $i$ and zero otherwise. We denote by $k_{i}=\sum_{j} a_{i j}$ the connectivity of site $i$ and by $j_{1}^{(i)}, j_{2}^{(i)}, \ldots, j_{k_{i}}^{(i)}$ its neighbourhood 
$\left(a_{i, j_{n}^{(i)}}=1\right)$. We consider only symmetric networks. We generate networks with $N$ nodes and $2 m N$ links, so that the average connectivity of each node is $\langle k\rangle=2 \mathrm{~m}$.

- Regular 1D: Nodes are arranged on a ring (periodic boundary condition). Any given node establishes a link with the $m$ closest nodes at its right. For instance for $m=2$, node 1 establishes a link with nodes 2 and 3 , node 2 with nodes 3 and 4 , and so on until nodes $N-2$ and $N-1$ establish a link with node 1.

- Random: Any node establishes $m$ links with randomly chosen nodes, avoiding self-loops and multiple links. The probability distribution of random networks is Poissonian, $P(k)=\frac{z^{k} e^{-z}}{k !}$, where $z=\langle k\rangle$.

- Scale-Free: we use a configurational model fixing also a cut-off $K$. First, at each node $i$ out of $N$ is assigned a connectivity $k_{i}$ draft from a power-law distribution $P(k)=A k^{-\gamma}, m \leq k \leq K$, with $A=(\gamma-1) /\left(m^{1-\gamma}-K^{1-\gamma}\right)$. Then links are connected at random avoiding self-loops and multiple links, and finally the total number of link is pruned in order to adjust the total number of links. This mechanism allow us to generate scale-free networks with a given exponent $\gamma$. We generally use $\gamma=2.1$.

Our multiplex networks are composed by two sub-networks called Physical and Information. We first generate the physical network by choosing one from regular, random or scale-free. Then we generate a Virtual network also chosen from the three benchmark networks, with same average connectivity $\langle k\rangle=2 \mathrm{~m}$. In order to construct the information network we overlap the real and the virtual ones and then, for each node, we prune its "physical" links with probability $q$ and its "virtual" ones with probability $1-q$. The overlap between the physical and the information networks is thus $1-q$. The information network is not symmetric.

This procedure allows to study the effects of the difference between $R$, where the epidemic spreading takes place, and $V$, which is the information network where actors become aware of the disease (i.e., over which they evaluate the perception of the risk of being infected).

\section{INFECTION MODEL AND MEAN-FIELD APPROXIMATION}

We assume that the probability that a site $i$ is infected by a neighbour $j$ is given by 1 ]

$$
u(s, k)=\tau \exp \left(-J \frac{s}{k_{i}}\right)
$$

where $\tau$ is the "bare" infection probability and $s$ is the number of infected neighbours. The idea is that the perception of the risk, modelled as the percentage of infected neighbours and modulated by the factor $J$ effectively lowers the infection probability (for instance because people takes more precautions). In the case of information networks, the perception is computed on the information neighbourhood, while the actual infection process takes places on the physical network.

It is possible to derive a simple mean-field approximation for the fixed- $k$ case. Denoting by $c$ the fraction of infected individuals at time $t$ and by $c^{\prime}$ those at time $t+1$, we have, considering a random network,

$$
c^{\prime}=\sum_{s=0}^{k}\left(\begin{array}{l}
k \\
s
\end{array}\right) c^{s}(1-c)^{k-s} p(s, k)
$$

where $p(s, k)$ is the probability of being infected if there are $s$ out of $k$ infected neighbours. The probability $p$ depends on $u$ as

$$
p(s, k)=1-[1-u(s, k)]^{s},
$$

since the infection processes are independent (although the infection probabilities are coupled by the "perception"dependent infection probability $q$, Eq. 11.

Near the threshold, the probability $u$ is small, and therefore we can approximate

$$
p(s, k) \simeq s u(s, k)=s \tau \exp \left(-J \frac{s}{k}\right) .
$$


Replacing into Eq. 1. we get

$$
c^{\prime}=\sum_{s=0}^{k}\left(\begin{array}{l}
k \\
s
\end{array}\right) c^{s}(1-c)^{k-s} s \tau \exp \left(-J \frac{s}{k}\right)
$$

and setting $a=\exp \left(-J \frac{s}{k}\right)$,

$$
c^{\prime}=\tau \sum_{s=0}^{k}\left(\begin{array}{l}
k \\
s
\end{array}\right) c^{s}(1-c)^{k-s} s a^{s},
$$

which gives

$$
c^{\prime}=\tau a k(c a+1-c)^{k-1} .
$$

The critical threshold $J_{c}$ corresponds to the stationary state $c^{\prime}=c$ in the limit $c \rightarrow 0$, i.e.,

$$
\tau=\frac{1}{k} \exp \left(\frac{J_{c}}{k}\right) ; \quad J_{c}=k \ln (k \tau) .
$$

This prediction is quite accurate: in Fig. 3 the comparison between Eq. 22) and actual simulations in reported for different values of $\langle k\rangle$ using random networks.

The analysis can be extended to non-homogeneous networks with connectivity distribution $P(k)$ like the scale-free ones. We can start analysing a node with connectivity $k$

$$
c_{k}^{\prime}=\sum_{s_{1}, s_{2}, \ldots, s_{k}=0}^{1} \sum_{j_{1}, j_{2}, \ldots, j_{k}=0}^{\infty} \prod_{i=1}^{k} C\left(j_{i}, k\right) I\left(s_{i}, j_{i}\right) T\left(k \mid s_{i}\right),
$$

where we denote with $i=1, \ldots, k$ the neighbours, $s_{i}=0,1$ is their state (healthy, infected) and $j_{i}$ their connectivity. $C(j, k)$ is the probability that a node with connectivity $j$ is attached to node with connectivity $k$, $I\left(s_{i}, j_{i}\right)$ is the probability that the neighbour $i$ is infected, and $T\left(k \mid s_{i}\right)$ is the probability that it transmits the infection to the node under investigation.

Clearly, $\sum_{j} C(j, k)=1$. We use symmetric networks, so $j C(j, k) P(j)=k C(k, j) P_{k}$ (detailed balance). For non-assortative networks, $C(j, k)$ does not depend on $k$, and summing over the detailed balance condition, $C(j, k)=j P(j) /\langle k\rangle$. The quantity $I\left(s_{i}, j_{i}\right)$ is simply $c_{j_{i}}^{s_{i}}\left(1-c_{j_{i}}\right)^{1-s_{i}}$ and $T\left(k \mid s_{i}\right)=1-(1-\tau \exp (-J s / k))^{s}$, where $s=\sum_{i} s_{i}$ (risk perception). Near the extinction, $\tau \exp (-J s / k)$ is small and we can approximate $T\left(k \mid s_{i}\right)=$ $s \tau \exp (-J s / k)$,

Summing up, we have

$$
\begin{aligned}
c_{k}^{\prime} & =\sum_{s_{1}, s_{2}, \ldots, s_{k}=0}^{1} \sum_{j_{1}, j_{2}, \ldots, j_{k}=0}^{\infty} \prod_{i=1}^{k} \frac{j_{i} P\left(j_{i}\right)}{\langle k\rangle} c_{j_{i}}^{s_{i}}\left(1-c_{j_{i}}\right)^{1-s_{i}} s \tau \exp \left(-J \frac{s}{k}\right), \\
& =\sum_{s_{1}, s_{2}, \ldots, s_{k}=0}^{1} s \tau \exp \left(-J \frac{s}{k}\right) \prod_{i=1}^{k} \sum_{j_{i}=0}^{\infty} \frac{j_{i} P\left(j_{i}\right)}{\langle k\rangle} c_{j_{i}}^{s_{i}}\left(1-c_{j_{i}}\right)^{1-s_{i}} .
\end{aligned}
$$

Since $s_{i}=0,1, c_{j_{i}}^{s_{i}}\left(1-c_{j_{i}}\right)^{1-s_{i}}$ is either $s_{i}$ or $1-s_{i}$. Let us define $\tilde{c}=\sum_{j} j c_{j} /\langle k\rangle$, and we get

$$
\begin{aligned}
c_{k}^{\prime} & =\sum_{s_{1}, s_{2}, \ldots, s_{k}=0}^{1} s \tau \exp \left(-J \frac{s}{k}\right) \prod_{i=1}^{k} \tilde{c}^{s_{i}}(1-\tilde{c})^{1-s_{i}}, \\
& =\sum_{s=0}^{k}\left(\begin{array}{c}
k \\
s
\end{array}\right) s \tau \exp \left(-J \frac{s}{k}\right) \tilde{c}^{s}(1-\tilde{c})^{k-s} \\
& =\tilde{c} k \tau \exp \left(\frac{-J s}{k}\right)\left(\tilde{c} \exp \left(-\frac{J}{k}\right)+1-\tilde{c}\right) .
\end{aligned}
$$


Near the epidemic threshold $\tilde{c} \rightarrow 0$, and

$$
\tilde{c}^{\prime}=\frac{1}{\langle k\rangle} \sum_{k} k c_{k}^{\prime} P(k)=\frac{\tau \tilde{c}}{\langle k\rangle} \sum_{k} k^{2} P(k) \exp \left(-\frac{J}{k}\right)
$$

The correspondence between $\tau_{c}$ and $J_{c}$ is therefore

$$
\tau_{c}\left(J_{c}\right)=\frac{\langle k\rangle}{\sum_{k} k^{2} P(k) \exp \left(-\frac{J_{c}}{k}\right)},
$$

which, for $J_{c}=0$, gives the usual relationship $\tau_{c}=\langle k\rangle /\left\langle k^{2}\right\rangle$, that for a sharply peaked $P(k)$ corresponds to Eq. (2).

By using a continuous approximation, it is possible to make explicit the relationship between $\tau$ and $J_{c}$ in the scale-free case. Eq. (3) becomes

$$
\tau_{c}\left(J_{c}\right)=\frac{\langle k\rangle}{\int_{m}^{K} k^{2} P(k) \exp \left(-\frac{J_{c}}{k}\right) \mathrm{d} k},
$$

Substituting, for the scale free case, $P(k)=A k^{-\gamma}$, where $A$ is the normalization constant so that $\int_{m}^{K} P(k)=1$

$$
A=\frac{\gamma-1}{m^{1-\gamma}-K^{1-\gamma}} \simeq(\gamma-1) m^{\gamma-1}
$$

if $K \gg m($ and $\gamma<3)$. We get

for $K \gg m$, and

$$
\langle k\rangle=\frac{\gamma-1}{\gamma-2} \cdot \frac{m^{2-\gamma}-K^{2-\gamma}}{m^{1-\gamma}-K^{1-\gamma}} \simeq \frac{\gamma-1}{\gamma-2} m
$$

$$
\tau_{c}\left(J_{c}\right)=J_{c}^{3-\gamma}\left[\Gamma\left(\gamma-3, \frac{J_{c}}{K}\right)-\Gamma\left(\gamma-3, \frac{J_{c}}{m}\right)\right],
$$

where $\Gamma(a, x)$ is the incomplete gamma function. Eq. (5) diverges for $K \rightarrow \infty$ and thus for infinite networks $J_{c}=0 \forall \tau$. However, real networks always have a cut-off (at least due to the finite number of nodes) [15]. For $J_{c}=0$ we recover the standard threshold

$$
\tau_{c}(0)=\frac{\gamma-3}{\gamma-2} \cdot \frac{m^{2-\gamma}-K^{2-\gamma}}{m^{3-\gamma}-K^{3-\gamma}} \simeq \frac{3-\gamma}{\gamma-2} \cdot \frac{m^{2-\gamma}}{K^{3-\gamma}}
$$

The problem of determining the epidemic threshold in finite-size scale-free networks was studied in a previous work [16]. The conclusions there is that even in finite-size networks the epidemics is hard to stop. Indeed, we find numerically that the epidemics always stops in finite scale-free networks although the required critical value of $J_{c}$ may be quite large.

In Fig. 4 the comparison between the mean-field prediction, Eq. (3), and actual simulations is shown, for three instances of a scale-free network generated with the same parameters. The theoretical prediction coincides with the simulations only for $J_{c}=0$. Moreover, although the simulation results seems to be sufficiently independent on the details of the generated networks, the theoretical prediction is quite sensible to them. The continuous approximation, Eq. (6) gives, for $m=2, K=300$ and $\gamma=2.4$, a value $\tau(0) \simeq 0.037$, quite different from the computed one $\tau(0) \simeq 0.08$.

\section{THE SELF-ORGANIZED PERCOLATION METHOD}

Here we show a self-organized percolation method that allow to obtain the critical value of the percolation parameter in a single run, for a given network. We consider a parallel SIS process, which is equivalent to a directed percolation problem where the directed direction is time. 


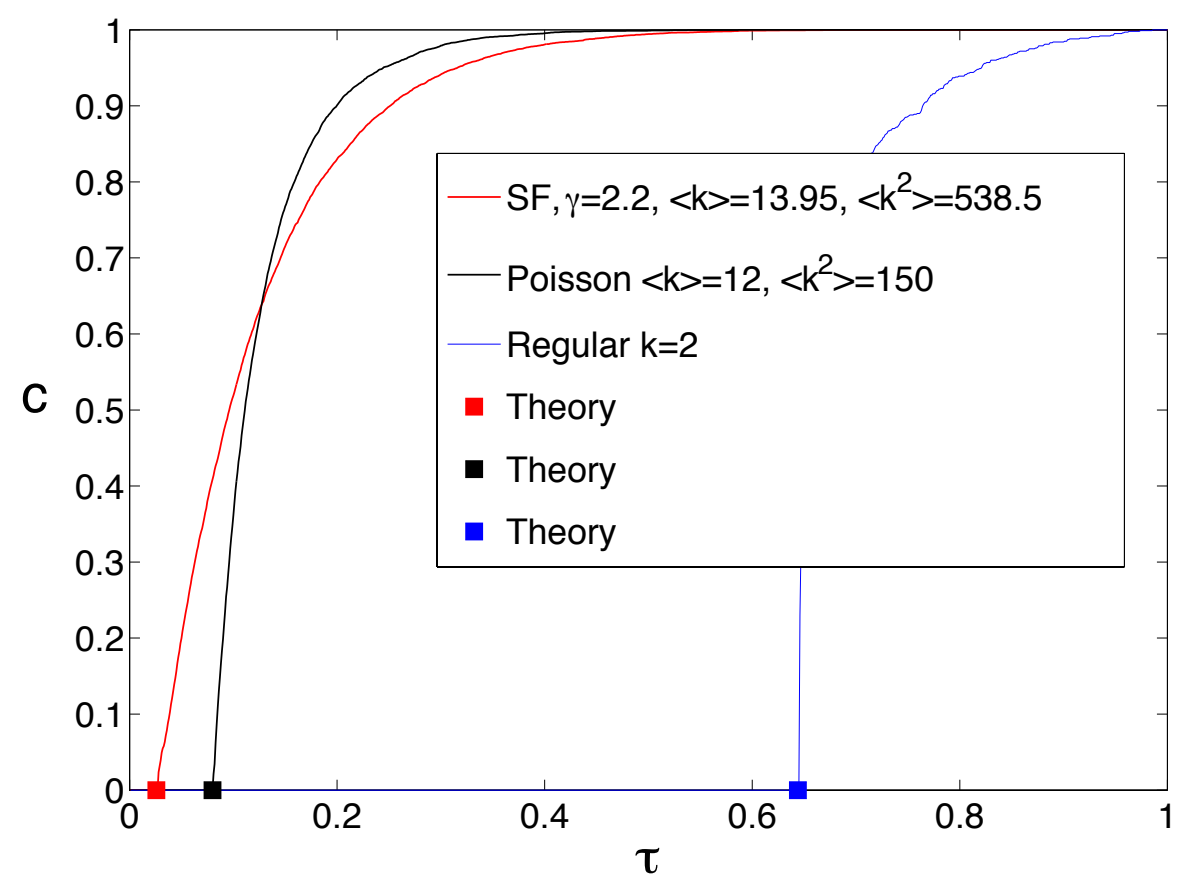

FiguRE 1. Asymptotic number of infected individuals $c$ versus the bare infectiousness probability $\tau$ for the SIS dynamics for different networks, $N=10000$.

Let us denote by $x_{i}(t)=0,1(0=$ healthy, $1=$ infected $)$, the percolating variable and by $p$ the control parameter (percolation probability).

\subsection{Direct percolation (simple infection)}

Considering $p$ is fixed, the stochastic evolution process for the network is defined as

$$
x_{i}(t+1)=\bigvee_{j=j_{1}^{(i)}, \ldots, j_{k_{i}}^{(i)}}\left[p>r_{i j}(t)\right] x_{j}(t)
$$

where $\bigvee$ represents the OR operator and the multiplication represents the AND. The square bracket represents the the truth function, $[\cdot]=1$ if "." is true, and zero otherwise. The quantity $r_{i j}(t)$ is a random number between 0 and 1 that varies with $i, j$ and $t$. We want to derive an equation for $p_{i}(t)$, which is the minimum value of $p$ for which $x_{i}(t)$ is infected. We can replace $x_{i}(t)$ by $\left[p>p_{i}(t)\right]$. Eq. 7 becomes:

$$
\left[p>p_{i}(t+1)\right]=\bigvee_{j=j_{1}^{(i)}, \ldots, j_{k_{i}}^{(i)}}\left[p>r_{i j}(t)\right]\left[p>p_{j}(t)\right]
$$

Now $[p>a][p>b]$ is equal to $[p>\max (a, b)]$ and $[p>a] \vee[p>b]$ is equal to $[p>\min (a, b)]$, therefore Eq. 8 becomes:

$$
\left[p>p_{i}(t+1)\right]=\left[p>\left(\underset{j=j_{1}^{(i)}, \ldots, j_{k_{i}}^{(i)}}{\operatorname{MAX}} \max \left(r_{i j}(t), p_{j}(t)\right)\right)\right],
$$




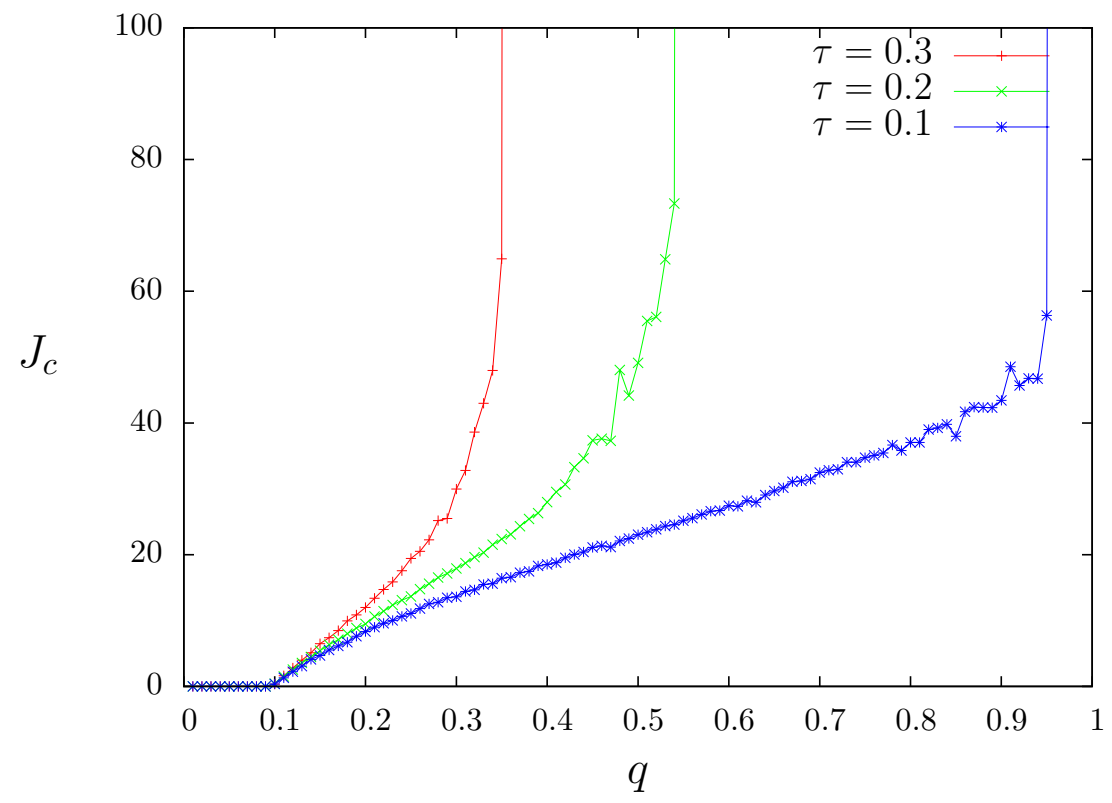

FiguRE 2. Critical precaution threshold $J_{c}$ versus the difference between the physical and the information network $q$ for some values of the bare infectiousness $\tau$ (from right to left $\tau=$ $0.1,0.2,0.3)$. Random physical network and scale-free virtual network, both with $\langle k\rangle=6$, $N=10000$.

and therefore we get the equations for the $p_{i}$ 's

$$
p_{i}(t+1)=\underset{j=j_{1}^{(i)}, \ldots, j_{k_{i}}^{(i)}}{\operatorname{MIN}} \max \left(r_{i j}(t), p_{j}(t)\right)
$$

Let assume that at time $t=0$ all sites are infected, so that $x_{i}(0)=1 \forall p$. We can alternatively write $p_{i}(0)=0$ (since the minimum value of $p$ for which $x_{i}(0)=1$ is one for sure. We can therefore iterate Eq. 10 and get the asymptotic distribution of $p_{i}$. The minimum of this distribution gives the critical value $p_{c}$ for which there is at least one percolating cluster with at least one "infected" site at large times. As usual, $t$ cannot be infinitely large for finite $N$ otherwise there will be surely a fluctuation that will bring the system into the absorbing (healthy $\left.x_{i}=0\right)$ configuration.

\subsection{Direct percolation with risk perception}

Now, let us apply the method to a more difficult problem, for which the percolation probability depends on the fraction of infected sites in the neighborhood (risk perception). As above, we define the infection probability $u$ as

$$
u(s, k)=\tau \exp \left(-J \cdot \frac{s}{k}\right)
$$

where $\tau$ is the bare infection probability, $s$ is the number of infected neighbours and $k$ is the node connectivity. In this case we want to find the minimum value of the parameter $J$ for which there is no spreading of the infection at large times. The quantity $[u>r]=[\tau \exp (-J s / k)>r]$ is equivalent to $[J<-(k / s) \ln (r / \tau)]$. 


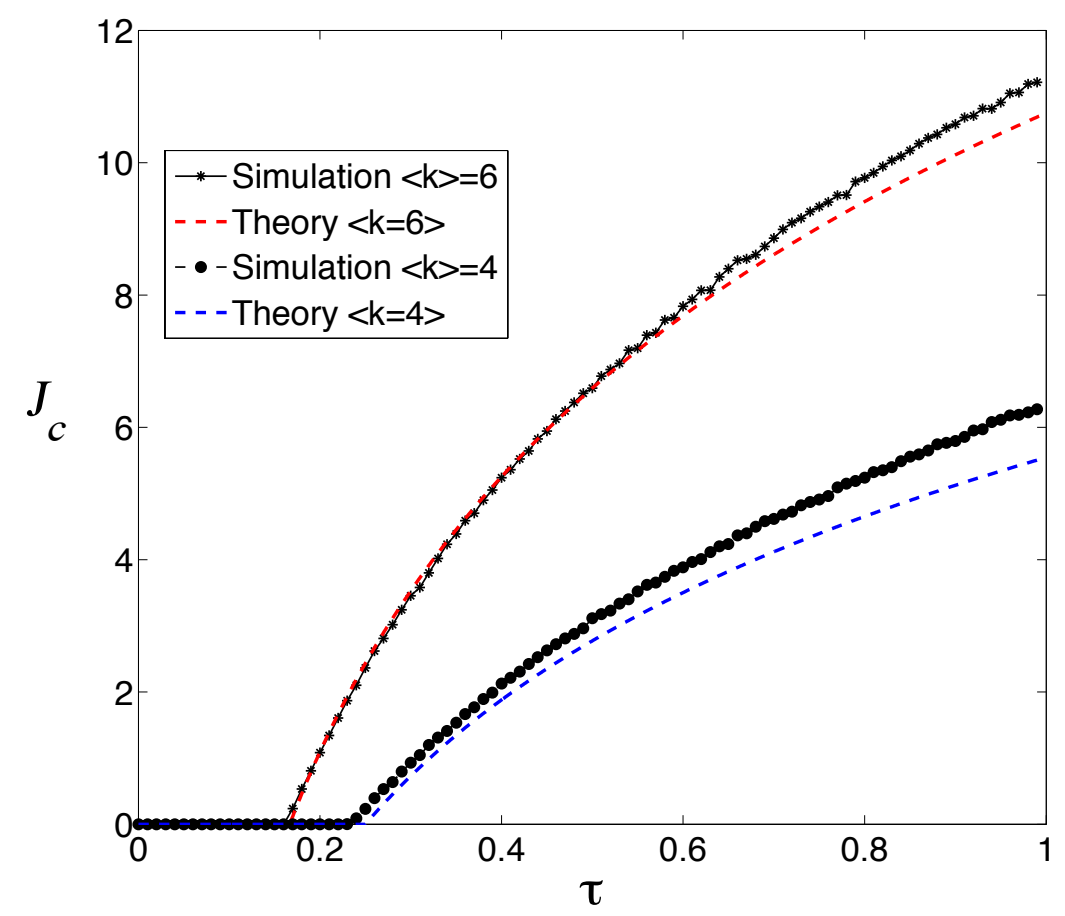

FiguRE 3. Comparison between mean field approximation and simulations for random networks with different values of $\langle k\rangle$.

Therefore Eq. (8) is replaced by

$$
\left[J<J_{i}(t+1)\right]=\bigvee_{j=j_{1}^{(i)}, \ldots, j_{k_{i}}^{(i)}}\left[J<-\frac{k_{i}}{s_{i}} \ln \left(\frac{r_{i j}(t)}{\tau}\right)\right]\left[J<J_{j}(t)\right]
$$

where

So

$$
s_{i} \equiv s_{i}(J)=\sum_{j=j_{1}^{(i)}, \ldots, j_{k_{i}}^{(i)}} x_{j}=\sum_{j=j_{1}^{(i)}, \ldots, j_{k_{i}}^{(i)}}\left[J_{j}(t) \geq J\right]
$$

and therefore

$$
\left[J<J_{i}(t+1)\right]=\bigvee_{j=j_{1}^{(i)}, \ldots, j_{k_{i}}^{(i)}}\left[J<-\frac{k_{i}}{s_{i}\left(J_{j}(t)\right)} \ln \left(\frac{r_{i j}(t)}{\tau}\right)\right]\left[J<J_{j}(t)\right]
$$

$$
J_{i}(t+1)=\operatorname{MAX}_{j=j_{1}^{(i)}, \ldots, j_{k_{i}}^{(i)}} \min \left(-\frac{k_{i}}{s_{i}\left(J_{j}(t)\right)} \ln \left(\frac{r_{i j}(t)}{\tau}\right), J_{j}(t)\right) .
$$

Analogously to the previous case, the critical value of $J_{c}$ is obtained by taking the maximum value of the $J_{i}(t)$ for some large (but finite) value of $t$.

\subsection{The self-organized percolation method for multiplex networks}

We can now turn to the problem of computing the critical value $J_{c}$ if the perception is computed on the information network that is partially different from the physical one. Here the perception of the importance 


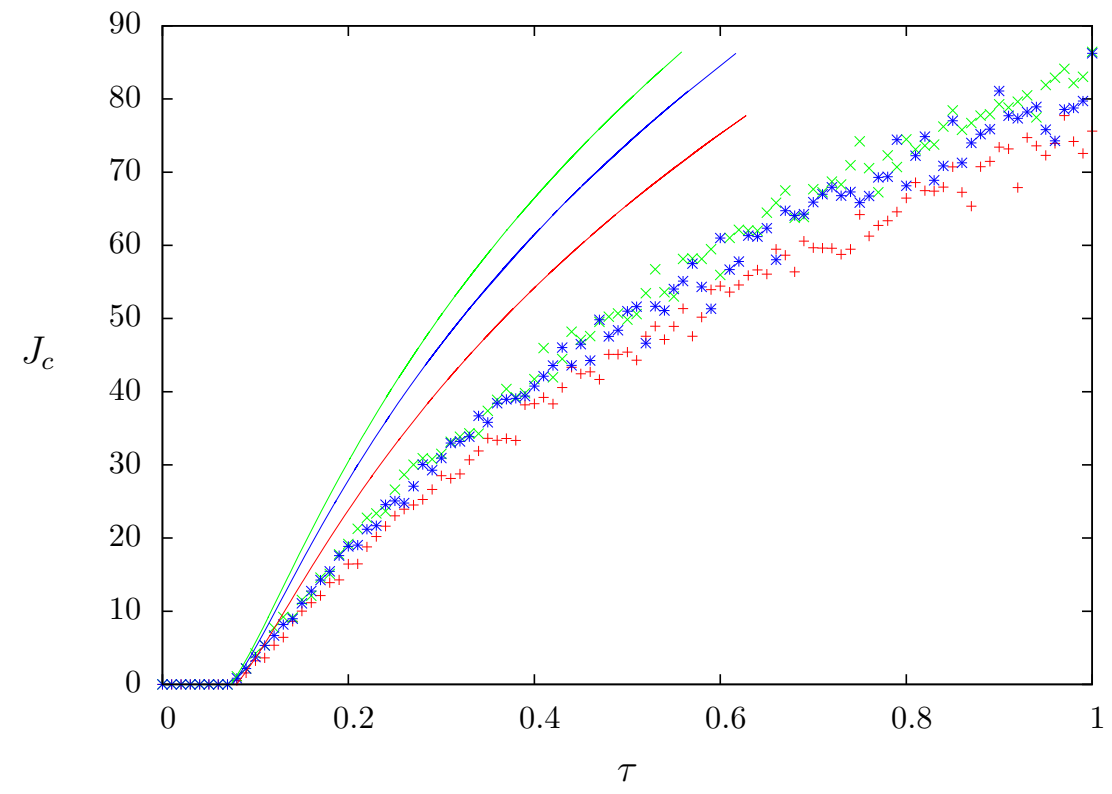

Figure 4. Comparison between the results of the mean field approximation and simulations for three instances of scale-free networks with $\gamma=2.4, N=10000, m=2$ and $K=300$. Although the simulations give similar results, the mean-field computations (that coincide with the simulation only for $J_{c}=0$ ) are very dependent on the details of the network.

of the infection, $\bar{s}_{i}$, is computed on the neighbours $\bar{j}^{(i)}$ on the information network. The perceived number of infected neighbours depends on how many of them, in the information network, have a value $J_{\bar{j}}$ larger than that computed in the physical network, i.e.,

$$
J_{i}(t+1)=\underset{j=j_{1}^{(i)}, \ldots, j_{k_{i}}^{(i)}}{\operatorname{MAX}} \min \left(-\frac{k_{i}}{\bar{s}_{i}\left(J_{j}\right)} \ln \left(\frac{r_{i j}(t)}{\tau}\right), J_{j}(t)\right),
$$

where

$$
\bar{s}_{i}(J)=\sum_{\bar{j}=\bar{j}_{1}^{(i)}, \ldots, \bar{j}_{\bar{k}_{i}}^{(i)}}\left[J_{\bar{j}} \geq J\right] .
$$

In other words: for any value of $J$ in the physical neighbourhood one computes how many neighbours of index $\bar{j}$ in the information network have $J_{\bar{j}} \geq J$. This is the perceived value of the risk.

\section{Results}

In this section we show the results of the self-organized percolation method in both single-layered and multiplex networks (with and without risk perception). For our experiments we fixed the size of the networks $N=1000$ and the percolation time $T=10000$.

\subsection{Percolation in single-layered networks}

We investigated the SIS dynamics over regular, Poisson and scale-free networks as shown in Fig. 1 in particular we evaluated the critical epidemic threshold values $p_{c} \equiv \tau_{c}$ for which there is at least one percolating clusters with at least one infected nodes. 


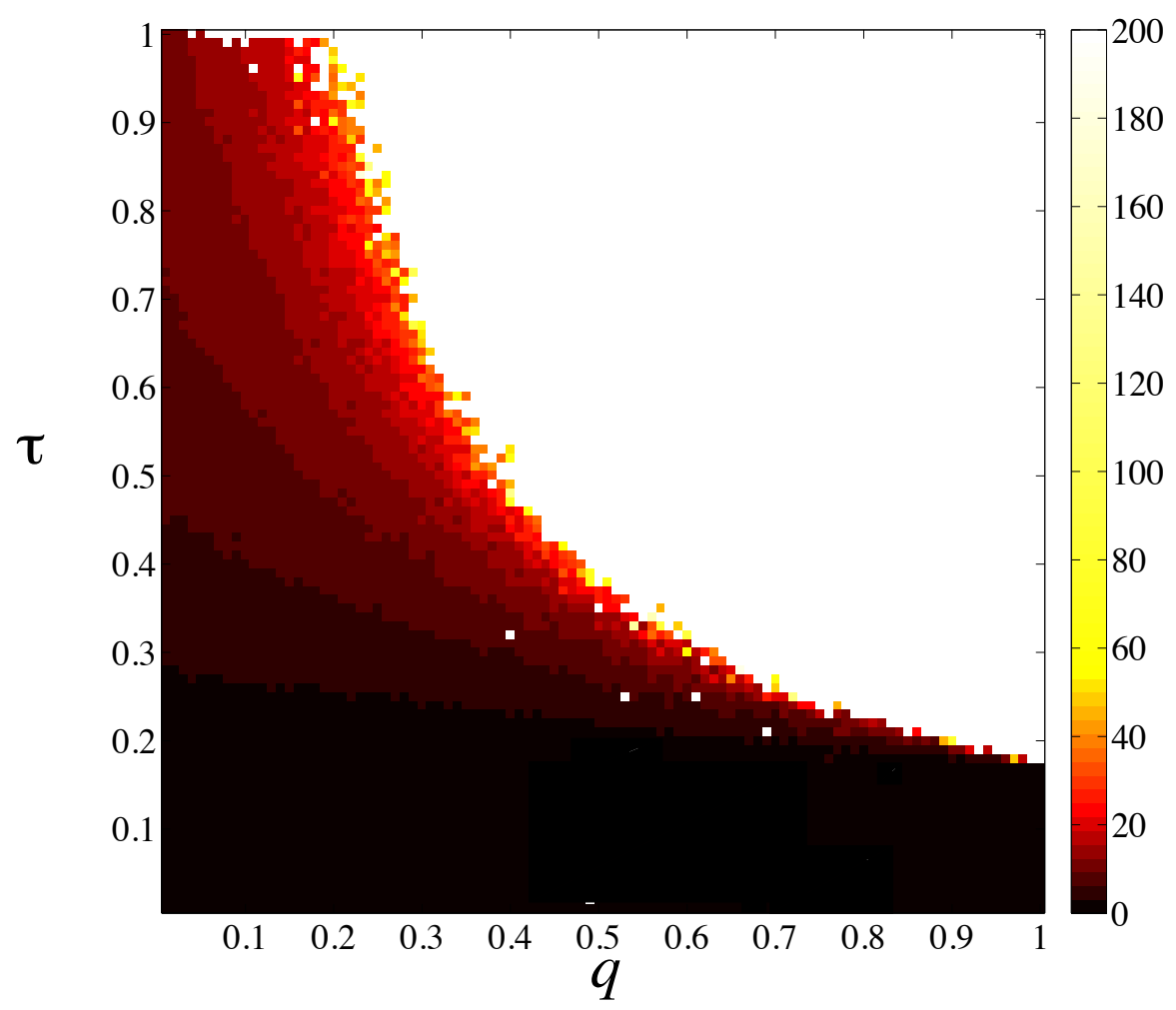

FiguRE 5. Critical precaution threshold $J_{c}$ (color code) as a function of the bare infectiousness $\tau$ and of the difference between the physical and the information network $q$. Here the physical and virtual networks are Poissonian (random) with $\langle k\rangle=6$ and $N=1000$. In the darker region there is always a value of $J_{c}$ able to stop the epidemics, while in the lighter region the epidemics cannot be stopped. The separation boundary is the stoppability frontier.

Considering a regular lattice with connectivity degree $k=2$, we found $\tau_{c} \simeq 0.6447$ which is compatible with the results of the bond percolation transition in the Domany-Kinzel model [17].

In the case of random networks with Poisson degree distributions the critical epidemic threshold $\tau_{c}=$ $\langle k\rangle /\left\langle k^{2}\right\rangle \simeq\langle k\rangle^{-1}$ if the distribution is sharp 18. Indeed, for Poisson network with $\langle k\rangle=12$ the self-organized percolation method gives $\tau_{c} \simeq 0.08 \simeq 1 / 12$.

For a scale-free network with $\langle k\rangle=13.95$ and $\left\langle k^{2}\right\rangle=538.5$ we get from simulations $\tau_{c} \simeq 0.026$, in agreement with the expected value.

\subsection{The effects of risk perception in SIS dynamics}

We investigate the effects of risk perception in a simple model of epidemic spreading. Here we show the results of the direct percolation method comparing the simulations with the theoretical formulation derived in Section 2

Our results are quite interesting if compared with the simple SIS dynamics (Fig. 1). Here we are able to stop the epidemic increasing the bare infection $\tau$ until $\tau=1$. Let us consider for instance the case of random networks with $\langle k\rangle=6$ in which we found a critical value of $\tau_{c}=0.165$ (Fig. 1). Above $\tau_{c}$, we need to adopt a precaution level $J>0$ in order to stop the spreading of the disease. The same consideration can be done also for the other scenarios. 


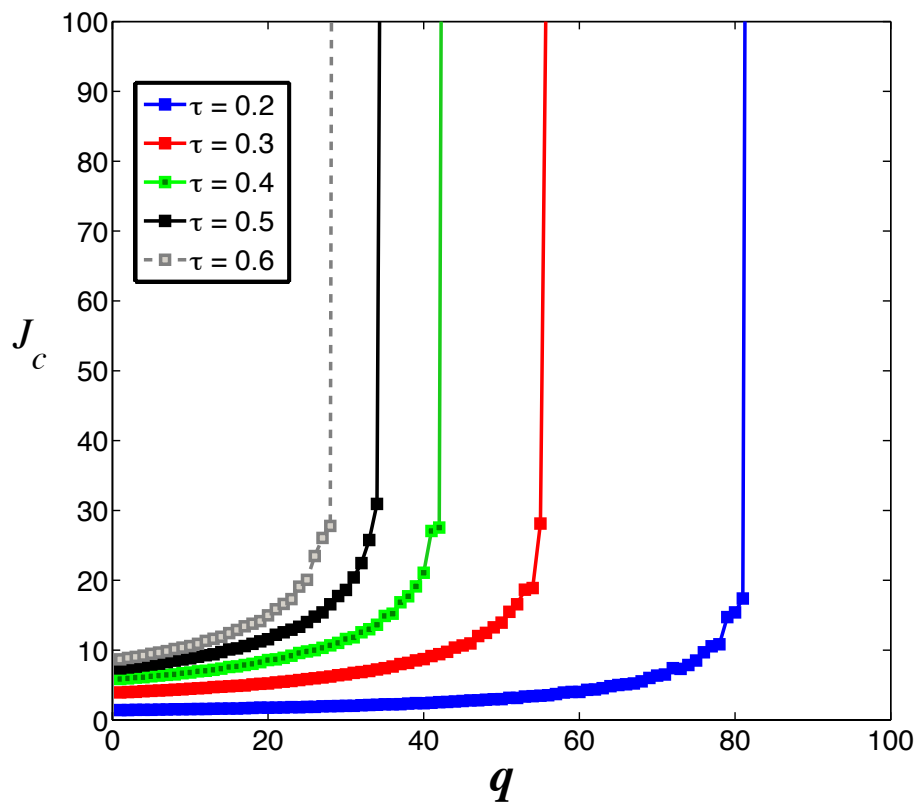

Figure 6. Critical precaution threshold $J_{c}$ versus the difference $q$ between the physical and the information networks for some values of the bare infectiousness $\tau$ (from right to left: $\tau=0.2,0.3, \ldots, 0.6)$. Random physical and virtual networks, both with $\langle k\rangle=6$ and $N=10000$.

\subsection{Multiplex risk perception}

The phase diagram for the risk-perception in modelling SIS dynamics in multiplex networks is reported in Fig. 5. The general shape of this phase diagram can be understood considering the continuous approximation, Eq. (4). A given node of connectivity $k$ is connected, in the information network, to a $(1-q) k$ physical neighbours, and to $q k$ virtual ones. At the threshold, the global fraction of infected sites is small. For the spreading of the epidemics, the important sites are those that have an infected physical neighbour. It might be assumed that the virtual neighbours, being uncorrelated with the physical ones, do not contribute at all to the risk perception. Among the physical neighbours, the fraction $k q$ replaced by virtual ones has become invisible, and so the perception decreases by a factor $q$. Replacing $J_{c}$ with $q J_{c}$ in Eq. (4) for the scale-free case one gets $\tau q^{3-\gamma}=$ const, and indeed the shape of the stoppability frontier of Fig. 5 resembles that of a generalized hyperbola.

The general trend is that, increasing the difference $q$ between the information network and the physical one it becomes harder to stop an epidemics. It is interesting to investigate this transition. As we can see in Fig. 2, this transition is quite sharp, especially for low values of $\tau$. A similar scenario holds for a mixture of random physical and random virtual networks, as shown in Fig. 6 .

\section{Conclusions}

We investigated the interplay between epidemic spreading and risk perception on multiplex networks, exploiting mean-field approximations and a self-organized method, that automatically gives the percolation threshold in just one simulation.

We considered two layers, one for the spreading of the information about the disease, and one for the actual spreading of the disease. The information level was considered proportional to the infection level in one's 
neighbourhood. However, this neighbourhood was composed by a mixture of actual physical contacts (that also contribute to effective risk of being infected) and by virtual contacts, extracted using various criteria but in general unrelated with physical neighbours. The news from the virtual network are mainly useless, carrying at most information about the average penetration of the infection in the population. In other words, we considered that people get infected by contacts in real life but often gather information from an information network, which may be quite different from the one of physical contacts. The information level, i.e., the risk perception, is considered affecting the probability of establishing an unprotected contact, thus lowering the effective invectiveness of the disease.

Our main conclusion is that the similarity between the physical and the information networks determines the possibility of stopping the infection for a sufficiently high precaution level: if the networks are too different there is no mean of avoiding the epidemics. Moreover, especially for low values of the bare infectiousness probability, the transition from a moderate infection level to a real outbreak occurs sharply without evident forerunners. This last observation remarks that, although the virtual world has indeed the advantage of allowing a fast diffusion of information, real epidemics still propagate in the physical world.

This is of particular importance for diseases diffused in marginalized parts of the population that may have little access to Internet, and that in any case are not exchanging information with people belonging to other social classes, but that may nonetheless be part of their "physical" neighborhood, for instance by commuting together, or meeting at work, etc. Just as an example, a person living in Europe or USA rarely considers the possibility of contracting tuberculosis, which is indeed rather a rare disease 19 but may cause sudden outbreaks in specific areas 20$]$.

\section{REFERENCES}

[1] F Bagnoli, P. Liò, and L. Sguanci. Risk perception in epidemic modeling. Phys. Rev. E, 76:061904, 2007.

[2] J. Ginsberg, M. Mohebbi, R. Patel, L. Brammer, M. Smolinski, and L. Brilliant. Detecting influenza epidemics using search engine query data. Nature, 457:1012-1014, 2009.

[3] D. Scanfeld, V. Scanfeld, and E. L. Larson. Dissemination of health information through social networks: Twitter and antibiotics. American Journal of Infection Control, 38(3):182 - 188, 2010.

[4] C. Chew and G. Eysenbach. Pandemics in the age of twitter: Content analysis of tweets during the 2009 h1n1 outbreak. PLoS ONE, 5(11):e14118, 112010.

[5] The State of the News Media The Pew Research Center's project for Excellence in Journalism, 2010.

[6] M. Kurant and P. Thiran. Layered complex networks. Phys. Rev. Lett., 96:138701, 2006.

[7] P. J. Mucha, T. Richardson, K. Macon, M.A. Porter, and J.-P. Onnela. Community structure in time-dependent, multiscale, and multiplex networks. Science, 328(5980):876-878, 2010.

[8] M. Szell, R. Lambiotte, and S. Thurner. Multirelational Organization of Large-scale Social Networks in an Online World. 2010.

[9] A. Arenas S. Lozano, X-P. Rodriguez. Evolution of cooperation in multiplex networks. Scientific reports, $2,2012$.

[10] G. Bianconi. Statistical mechanics of multiplex networks: Entropy and overlap. Phys. Rev. E, 87:062806, 2013.

[11] S. V. Buldyrev, R. Parshani, G. Paul, H. E. Stanley, and S. Havlin. Catastrophic cascade of failures in interdependent networks. Nature, 464(7291):1025-1028, 2010.

[12] J. Gao, S. V. Buldyrev, H. E. Stanley, and S. Havlin. Networks formed from interdependent networks. Nat Phys, 8(1):40-48, 2012.

[13] C. Granell, S. Gómez, and A. Arenas. Dynamical interplay between awareness and epidemic spreading in multiplex networks. Phys. Rev. Lett., 111:128701, Sep 2013.

[14] F. Bagnoli, P. Palmerini, and R. Rechtman. Algorithmic mapping from criticality to self-organized criticality. Phys. Rev. E, 55:3970-3976, Apr 1997.

[15] S. N. Dorogovtsev and J.F.F. Mendes. Evolution of networks. Advances in Physics, 51:1079-1187, 2002.

[16] R. Pastor-Satorras and A. Vespignani. Epidemic dynamics in finite size scale-free networks. Physical Review E, 65(035108), 2002.

[17] E. Domany and W. Kinzel. Equivalence of cellular automata to ising models and directed percolation. Phys. Rev. Lett., 53:311-314, 1984.

[18] R. Pastor-Satorras and A. Vespignani. Epidemic spreading in scale-free networks. Phys. Rev. Lett., 86:3200-3203, 2001.

[19] World Health Organization. Global tubercolosis report, 2013.

[20] M. Faccini, L. R. Codecasa, G. Ciconali, S. Cammarata, C.R. Borriello, C. De Gioia, A. Za, A. F. Marino, V. Vighi, M. Ferrarese, G. Gesu, E. Mazzola, and S. Castaldi. Tuberculosis outbreak in a primary school, Milan, Italy. Emerg. Infect. Dis. [Internet], Mar. 2013. 